\title{
GLOBAL REGULARITY FOR SOLUTIONS OF THE NAVIER-STOKES EQUATION SUFFICIENTLY CLOSE TO BEING EIGENFUNCTIONS OF THE LAPLACIAN
}

\author{
EVAN MILLER
}

(Communicated by Ryan Hynd)

\begin{abstract}
In this paper, we will prove a new, scale critical regularity criterion for solutions of the Navier-Stokes equation that are sufficiently close to being eigenfunctions of the Laplacian. This estimate improves previous regularity criteria requiring control on the $\dot{H}^{\alpha}$ norm of $u$, with $2 \leq \alpha<\frac{5}{2}$, to a regularity criterion requiring control on the $\dot{H}^{\alpha}$ norm multiplied by the deficit in the interpolation inequality for the embedding of $\dot{H}^{\alpha-2} \cap \dot{H}^{\alpha} \hookrightarrow \dot{H}^{\alpha-1}$. This regularity criterion suggests, at least heuristically, the possibility of some relationship between potential blowup solutions of the Navier-Stokes equation and the Kolmogorov-Obhukov spectrum in the theory of turbulence.
\end{abstract}

\section{INTRODUCTION}

The Navier-Stokes equation is one of the fundamental equations of fluid mechanics. For an incompressible fluid, where the density of the fluid in question is constant, the Navier-Stokes equation with no external forces is given by

$$
\begin{aligned}
\partial_{t} u-\Delta u+P_{d f}((u \cdot \nabla) u) & =0, \\
\nabla \cdot u & =0,
\end{aligned}
$$

where $u \in \mathbb{R}^{3}$ is the velocity of the fluid and $P_{d f}$ is the projection onto the space of divergence free vector fields. We have also taken the viscosity to be 1 , which we can do without loss of generality, because it is equivalent up to rescaling. In his foundational paper on the subject, Leray proved the global existence of weak solutions to the Navier-Stokes equation satisfying an energy inequality [18; however, such solutions are not known to be either smooth or unique. This is because the bounds from the energy inequality are both supercritical with respect to scaling. Smooth solutions of the Navier-Stokes equation with initial data in $H^{1}$ must satisfy an energy equality, stating that for all $t>0$,

$$
\frac{1}{2}\|u(\cdot, t)\|_{L^{2}}^{2}+\int_{0}^{t}\|\nabla u(\cdot, \tau)\|_{L^{2}}^{2} \mathrm{~d} \tau=\frac{1}{2}\left\|u^{0}\right\|_{L^{2}}^{2} .
$$

The Navier-Stokes equation is invariant under the rescaling,

$$
u^{\lambda}(x, t)=\lambda u\left(\lambda x, \lambda^{2} t\right)
$$

Received by the editors April 20, 2020, and, in revised form, August 3, 2020, and September 26, 2020.

2020 Mathematics Subject Classification. Primary 35Q30. 
for all $\lambda>0$, and it is a simple calculation to check that the bounds on $u$ in $L_{t}^{\infty} L_{x}^{2}$ and $L_{t}^{2} \dot{H}_{x}^{1}$ from the energy equality (1.1) are supercritical with respect to the rescaling (1.2).

The only results presently available guaranteeing regularity for the Navier-Stokes equation with general, arbitrarily large initial data, require control of some scale critical quantity. Ladyzhenskaya [17, Prodi [22, and Serrin [23, proved that if a smooth solution of the Navier-Stokes equation blows up in finite time $T_{\max }<+\infty$, then for all $3<q \leq+\infty, \frac{2}{p}+\frac{3}{q}=1$,

$$
\int_{0}^{T_{\max }}\|u(\cdot, t)\|_{L^{q}}^{p} \mathrm{~d} t=+\infty .
$$

It is straightforward to check that $L_{t}^{p} L_{x}^{q}$ is scale critical with respect to the rescaling (1.2), when $\frac{2}{p}+\frac{3}{q}=1$. This result was then extended by Escauriaza, Seregin, and Šverák 10 to the endpoint case $p=+\infty, q=3$. They proved that if a smooth solution $u$ of the Navier-Stokes equation blows up in finite time $T_{\max }<+\infty$, then

$$
\limsup _{t \rightarrow T_{\max }}\|u(\cdot, t)\|_{L^{3}\left(\mathbb{R}^{3}\right)}=+\infty .
$$

There have been a number of generalizations of the Ladyzhenskaya-Prodi-Serrin regularity criterion, including scale critical component reduction results involving the vorticity [3], two components of the vorticity [5], the derivative in just one direction, $\frac{\partial u}{\partial x_{i}}$ [16], and involving only one component, $u_{j}$ [6, 7]. The LadyzhenskayaProdi-Serrin regularity criterion has also been generalized to endpoint Besov spaces [8, 14, 15, while the Escauriaza-Seregin-Šverák regularity criteriona has been generalized to all non-endpoint Besov spaces 1, 2, 12.

In this paper, we will generalize the Ladyzhenskaya-Prodi-Serrin regularity criterion to solutions of the Navier-Stokes equation that are close to being eigenfunctions of the Laplacian. One tool we will use is the notion of mild solutions, which was developed by Kato and Fujita [1].

Definition 1.1. Suppose $u \in C\left([0, T) ; \dot{H}^{1}\left(\mathbb{R}^{3}\right)\right), \nabla \cdot u=0$. Then $u$ is a mild solution to the Navier-Stokes equation if for all $0<t<T$,

$$
u(\cdot, t)=e^{t \Delta} u^{0}+\int_{0}^{t} e^{(t-\tau) \Delta} P_{d f}(-(u \cdot \nabla) u)(\cdot, \tau) \mathrm{d} \tau,
$$

where $e^{t \Delta}$ is the heat semi-group operator given by convolution with the heat kernel; that is to say, $e^{t \Delta} u^{0}$ is the solution of the heat equation after time $t$, with initial data $u^{0}$.

Fujita and Kato proved the local existence in time of mild solutions to the NavierStokes equation for all initial data in $\dot{H}^{1}\left(\mathbb{R}^{3}\right)$. Our results for solutions of the Navier-Stokes equation sufficiently close to being eigenfunctions of the Laplacian will be proven in terms of $H^{1}$ mild solutions. We will also define, for all $\alpha>-\frac{3}{2}$, the homogeneous Sobolev space $\dot{H}^{\alpha}\left(\mathbb{R}^{3}\right)$, which is a Hilbert space for $-\frac{3}{2}<\alpha<\frac{3}{2}$, as the space with the norm

$$
\begin{aligned}
\|f\|_{\dot{H}^{\alpha}}^{2} & =\left\|(-\Delta)^{\frac{\alpha}{2}} f\right\|_{L^{2}}^{2} \\
& =\int_{\mathbb{R}^{3}}(2 \pi|\xi|)^{2 \alpha}|\hat{f}(\xi)|^{2} \mathrm{~d} \xi,
\end{aligned}
$$


and the inhomogeneous Hilbert space $H^{\alpha}\left(\mathbb{R}^{3}\right)$ as the space with the norm

$$
\|f\|_{H^{\alpha}}^{2}=\int_{\mathbb{R}^{3}}\left(1+4 \pi^{2}|\xi|^{2}\right)^{\alpha}|\hat{f}(\xi)|^{2} \mathrm{~d} \xi
$$

while further noting that for all $\alpha>0$,

$$
H^{\alpha}=L^{2} \cap \dot{H}^{\alpha} .
$$

For solutions of the Navier-Stokes equation, we call $\frac{1}{2}\|u(\cdot, t)\|_{L^{2}}^{2}$ the energy and $\frac{1}{2}\|u(\cdot, t)\|_{\dot{H}^{1}}^{2}$ the enstrophy. With mild solutions and the relevant Sobolev spaces defined, we can now state the main theorem of this paper.

Theorem 1.2. Suppose $u \in C\left(\left[0, T_{\max }\right) ; H^{1}\right)$ is a mild solution of the NavierStokes equation, and suppose $\frac{6}{5}<q \leq 3, \frac{2}{p}+\frac{3}{q}=3$. Then for all $0<t<T_{\max }$

$$
\|\nabla u(\cdot, t)\|_{L^{2}}^{2} \leq\left\|\nabla u^{0}\right\|_{L^{2}}^{2} \exp \left(C_{q} \int_{0}^{t} \inf _{\lambda \in \mathbb{R}}\|-\Delta u-\lambda u\|_{L^{q}}^{p} \mathrm{~d} \tau\right),
$$

where $C_{q}>0$ depends only on $q$. In particular, if $T_{\max }<+\infty$ then

$$
\int_{0}^{T_{\max }} \inf _{\lambda \in \mathbb{R}}\|-\Delta u-\lambda u\|_{L^{q}}^{p} \mathrm{~d} t=+\infty .
$$

An eigenfunction of the Laplacian satisfies the equation,

$$
-\Delta u-\lambda u=0 \text {. }
$$

There are no nonzero eigenfunctions of the Laplacian in $H^{1}\left(\mathbb{R}^{3}\right)$, because if $-\Delta u=$ $\lambda u$, that would require its Fourier transform to be supported on a set of measure zero, specifically the set

however, the quantity

$$
\left\{\xi \in \mathbb{R}^{3}: 4 \pi^{2}|\xi|^{2}=\lambda\right\}
$$

$$
\int_{0}^{t} \inf _{\lambda \in \mathbb{R}}\|-\Delta u-\lambda u\|_{L^{q}}^{p} \mathrm{~d} \tau
$$

is nonetheless a scale critical measure of how close a solution is to being an eigenfunction of the Laplacian. This quantity is scale invariant because this infimum scales the same way as $-\Delta u$, the quantity with no parameter in the infimum, and we can see from the scale invariance (1.2), that $-\Delta u$ has the scale invariance

$$
-\Delta u^{\lambda}(x, t)=-\lambda^{3} \Delta u\left(\lambda x, \lambda^{2} t\right) .
$$

It is a simple calculation to observe that when $\frac{2}{p}+\frac{3}{q}=3$, the space $L_{t}^{p} L_{x}^{q}$ is invariant under this rescaling.

It is also important to note here that the infimum in the regularity criterion is taken at each time, and so $\lambda$ can vary in time. It is only necessary that $u$ be sufficiently close to being an eigenfunction for some eigenvalue at each time; it is not necessary that $u$ be close to being an eigenfunction for a fixed eigenvalue. Because the infimum is inside the integral rather than outside, this rules out a wider range of blowup conditions.

We do not have a nice expression for the quantity

$$
\inf _{\lambda \in \mathbb{R}}\|-\Delta u-\lambda u\|_{L^{q}}
$$

in general $L^{q}$ spaces, however in $L^{2}$ we can use the Hilbert space structure to calculate this quantity explicitly, which allows us to obtain the following result. 
Corollary 1.3. Suppose $u \in C\left(\left[0, T_{\max }\right) ; H^{1}\right)$ is a mild solution of the NavierStokes equation. Then for all $0<t<T_{\max }$

$$
\|\nabla u(\cdot, t)\|_{L^{2}}^{2} \leq\left\|\nabla u^{0}\right\|_{L^{2}}^{2} \exp \left(C_{2} \int_{0}^{t}\|-\Delta u\|_{L^{2}}^{\frac{4}{3}}\left(1-\frac{\|\nabla u\|_{L^{2}}^{4}}{\|u\|_{L^{2}}^{2}\|-\Delta u\|_{L^{2}}^{2}}\right)^{\frac{2}{3}} \mathrm{~d} \tau\right),
$$

where $C_{2}>0$ is taken as in Theorem 1.2. In particular, if $T_{\max }<+\infty$ then

$$
\int_{0}^{T_{\max }}\|-\Delta u\|_{L^{2}}^{\frac{4}{3}}\left(1-\frac{\|\nabla u\|_{L^{2}}^{4}}{\|u\|_{L^{2}}^{2}\|-\Delta u\|_{L^{2}}^{2}}\right)^{\frac{2}{3}} \mathrm{~d} t=+\infty .
$$

More generally, we can use the Sobolev embedding of $\dot{H}^{\beta} \hookrightarrow L^{q}$ to generalize Corollary 1.3 in terms of homogeneous Sobolev spaces.

Corollary 1.4. Suppose $u \in C\left(\left[0, T_{\max }\right) ; H^{1}\right)$ is a mild solution of the NavierStokes equation, and suppose $2 \leq \alpha \leq \frac{5}{2}, \alpha=\frac{1}{2}+\frac{2}{p}$. Then for all $0<t<T_{\max }$

$$
\|\nabla u(\cdot, t)\|_{L^{2}}^{2} \leq\left\|\nabla u^{0}\right\|_{L^{2}}^{2} \exp \left(\tilde{C}_{\alpha} \int_{0}^{t}\|u\|_{\dot{H}^{\alpha}}^{p}\left(1-\frac{\|u\|_{\dot{H}^{\alpha-1}}^{4}}{\|u\|_{\dot{H}^{\alpha-2}}^{2}\|u\|_{\dot{H}^{\alpha}}^{2}}\right)^{\frac{p}{2}} \mathrm{~d} \tau\right),
$$

where $\tilde{C}_{\alpha}>0$ depends only on $\alpha$. In particular, if $T_{\max }<+\infty$ then

$$
\int_{0}^{T_{\max }}\|u\|_{\dot{H}^{\alpha}}^{p}\left(1-\frac{\|u\|_{\dot{H}^{\alpha-1}}^{4}}{\|u\|_{\dot{H}^{\alpha-2}}^{2}\|u\|_{\dot{H}^{\alpha}}^{2}}\right)^{\frac{p}{2}} \mathrm{~d} t=+\infty .
$$

Note that the scaling relation between $\alpha$ and $p$ can alternatively be expressed by

$$
p=\frac{2}{\alpha-\frac{1}{2}}
$$

We will note here that the $\alpha=2$ case of Corollary 1.4, is precisely Corollary 1.3. For $2<\alpha \leq \frac{5}{2}$, Corollary 1.4 requires that we use the fractional Sobolev inequality to bound the infimum in $L^{q}$ by an infimum in the appropriate homogeneous Hilbert space, which can be calculated explicitly. We will show that

$$
\begin{aligned}
\inf _{\lambda \in \mathbb{R}}\|-\Delta u-\lambda u\|_{L^{q}}^{2} & \leq C \inf _{\lambda \in \mathbb{R}}\|-\Delta u-\lambda u\|_{\dot{H}^{\alpha-2}}^{2} \\
& =C\|u\|_{\dot{H}^{\alpha}}^{2}\left(1-\frac{\|u\|_{\dot{H}^{\alpha-1}}^{4}}{\|u\|_{\dot{H}^{\alpha-2}}^{2}\|u\|_{\dot{H}^{\alpha}}^{2}}\right),
\end{aligned}
$$

where $\alpha-2=\frac{3}{2}-\frac{3}{q}$.

We will also note that without the term $\left(1-\frac{\|u\|_{H^{\alpha-1}}^{4}}{\|u\|_{\dot{H}^{\alpha}-2}^{2}\|u\|_{\dot{H}^{\alpha}}^{2}}\right)^{\frac{p}{2}}$ Corollary 1.4 is an immediate corollary of a variant of the Ladyzhenskaya-Prodi-Serrin regularity criterion. Corollary 1.4 shows that our regularity criterion for solutions of the Navier-Stokes equation sufficiently close to being eigenfunctions of the Laplacian measures the deficit in the interpolation inequality for the embedding

$$
\dot{H}^{\alpha-2} \cap \dot{H}^{\alpha} \hookrightarrow \dot{H}^{\alpha-1},
$$

which states that

$$
\|f\|_{\dot{H}^{\alpha-1}}^{2} \leq\|f\|_{\dot{H}^{\alpha}}\|f\|_{\dot{H}^{\alpha-2}},
$$


where the constant 1 is sharp but not attained, because there are no nonzero eigenfunctions of the Laplacian in $\dot{H}^{\alpha-1}\left(\mathbb{R}^{3}\right)$. When the inequality (1.4) is close to being saturated, then the quantity

$$
\left(1-\frac{\|u\|_{\dot{H}^{\alpha-1}}^{4}}{\|u\|_{\dot{H}^{\alpha-2}}^{2}\|u\|_{\dot{H}^{\alpha}}^{2}}\right)^{\frac{p}{2}}
$$

will be small, so Corollary 1.4 limits the extent to which blowup solutions can saturate the interpolation inequality (1.4).

We will also prove that finite-time blowup solutions cannot concentrate on arbitrarily narrow bands in Fourier space, supported between an inner radius of $R_{1}(t)$ and an outer radius of $R_{2}(t)$, with the ratio $\frac{R_{1}(t)}{R_{2}(t)} \rightarrow 1$ arbitrarily quickly as $t \rightarrow T_{\max }$ relative to the size of $\|u(\cdot, t)\|_{\dot{H}^{\alpha}}^{p}$.

Corollary 1.5. Suppose $u \in C\left(\left[0, T_{\max }\right) ; H^{1}\right)$ is a mild solution of the NavierStokes equation, and suppose for all $0<t<T_{\max }$

$$
\operatorname{supp} \hat{u}(t) \subset\left\{\xi \in \mathbb{R}^{3}: R_{1}(t) \leq|\xi| \leq R_{2}(t)\right\} .
$$

Let $2 \leq \alpha \leq \frac{5}{2}, \alpha=\frac{1}{2}+\frac{2}{p}$. Then for all $0<t<T_{\max }$

$$
\|\nabla u(\cdot, t)\|_{L^{2}}^{2} \leq\left\|\nabla u^{0}\right\|_{L^{2}}^{2} \exp \left(\tilde{C}_{\alpha} \int_{0}^{t}\|u\|_{\dot{H}^{\alpha}}^{p}\left(1-\frac{R_{1}(\tau)^{4}}{\left.R_{2}(\tau)\right)^{4}}\right)^{\frac{p}{2}} \mathrm{~d} \tau\right),
$$

where $\tilde{C}_{\alpha}>0$ depends only on $\alpha$. In particular, if $T_{\max }<+\infty$ then

$$
\int_{0}^{T_{\max }}\|u\|_{\dot{H}^{\alpha}}^{p}\left(1-\frac{R_{1}(t)^{4}}{\left.R_{2}(t)\right)^{4}}\right)^{\frac{p}{2}} \mathrm{~d} t=+\infty .
$$

There are a number of previous results for Navier-Stokes regularity criteria involving frequency localization in Fourier space [4,9, 20]. There is not enough space to discuss these results in detail here, as stating the main theorems would require us to define a number of objects from Littlewood-Paley theory, but we will note that the regularity criterion in [9] has an explicit connection with the Kolmogorov scaling in turbulence. Corollary 1.5 can also be seen as providing a heuristic connection between the regularity criterion in Theorem 1.2 and the Kolmogorov phenomenological theory of turbulence. Corollary[1.5] shows that solutions supported on narrow bands in Fourier space are not good candidates for finite-time blowup; this is consistent with the Kolmogorov-Obukhov phenomenology of turbulence [13,21, which stipulates that turbulent flows cannot localize around a small number of frequencies, specifically that the energy spectrum for turbulent flows has a decay in Fourier space on the order of $|\xi|^{-\frac{5}{3}}$ in the inertial range.

\section{Proofs of the Results}

Before beginning the proof of the main theorem, we will first need to establish an identity for the growth of enstrophy related to eigenfunctions of the Laplacian.

Lemma 2.1. Suppose $u \in C\left(\left[0, T_{\max }\right) ; H^{1}\right)$ is a mild solution of the Navier-Stokes equation. Then for all $0<t<T_{\max }$, and for all $\lambda \in \mathbb{R}$

$$
\frac{\mathrm{d}}{\mathrm{d} t} \frac{1}{2}\|\nabla u(\cdot, t)\|_{L^{2}}^{2}=-\|\Delta u\|_{L^{2}}^{2}-\langle-\Delta u-\lambda u,(u \cdot \nabla) u\rangle .
$$


Proof. It is easy to see from the Navier-Stokes equation that

$$
\frac{\mathrm{d}}{\mathrm{d} t} \frac{1}{2}\|\nabla u(\cdot, t)\|_{L^{2}}^{2}=-\|-\Delta u\|_{L^{2}}^{2}-\langle-\Delta u,(u \cdot \nabla) u\rangle .
$$

We know that $u \in H^{1}$, and therefore we have sufficient regularity to integrate by parts, using the condition $\nabla \cdot u=0$, to conclude that

$$
\langle(u \cdot \nabla) u, u\rangle=-\langle u,(u \cdot \nabla) u\rangle=0 .
$$

Because we know that for all $\lambda \in \mathbb{R}$

$$
\langle\lambda u,(u \cdot \nabla) u\rangle=0,
$$

we can conclude that for all $\lambda \in \mathbb{R}$,

$$
\frac{\mathrm{d}}{\mathrm{d} t} \frac{1}{2}\|\nabla u(\cdot, t)\|_{L^{2}}^{2}=-\|\Delta u\|_{L^{2}}^{2}-\langle-\Delta u-\lambda u,(u \cdot \nabla) u\rangle .
$$

This completes the proof.

One of the other main ingredients in our proof will be the fractional Sobolev inequality, which is stated below.

Theorem 2.2. Suppose $0<s<\frac{3}{2}$, and $\frac{1}{q}=\frac{1}{2}-\frac{s}{3}$. Then for all $f \in \dot{H}^{s}\left(\mathbb{R}^{3}\right)$,

$$
\|f\|_{L^{q}} \leq C_{s}\|f\|_{\dot{H}^{s}}
$$

where

$$
C_{s}=2^{-\frac{s}{3}} \pi^{-\frac{2}{3} s}\left(\frac{\Gamma\left(\frac{3}{2}-s\right)}{\Gamma\left(\frac{3}{2}+s\right)}\right)^{\frac{1}{2}}
$$

Note that the scaling relation between the parameters $q$ and $s$ can be stated equivalently as

$$
s=\frac{3}{2}-\frac{3}{q} .
$$

The Sobolev inequality was first proven by Sobolev [24] in the case where $s=1$. The sharp version of this inequality was proven by Talenti 25] in the case where $s=1$, and the general sharp version of this inequality with $0<s<\frac{3}{2}$ was proven by Lieb [19. With these results established, we can now prove Theorem 1.2, which is restated here for the reader's convenience.

Theorem 2.3. Suppose $u \in C\left(\left[0, T_{\max }\right) ; H^{1}\right)$ is a mild solution of the NavierStokes equation, and suppose $\frac{6}{5}<q \leq 3, \frac{2}{p}+\frac{3}{q}=3$. Then for all $0<t<T_{\max }$

$$
\|\nabla u(\cdot, t)\|_{L^{2}}^{2} \leq\left\|\nabla u^{0}\right\|_{L^{2}}^{2} \exp \left(C_{q} \int_{0}^{t} \inf _{\lambda \in \mathbb{R}}\|-\Delta u-\lambda u\|_{L^{q}}^{p} \mathrm{~d} \tau\right),
$$

where $C_{q}>0$ depends only on $q$. In particular, if $T_{\max }<+\infty$ then

$$
\int_{0}^{T_{\max }} \inf _{\lambda \in \mathbb{R}}\|-\Delta u-\lambda u\|_{L^{q}}^{p} \mathrm{~d} t=+\infty .
$$

Proof. We know that if $T_{\max }<+\infty$, then

$$
\lim _{t \rightarrow T_{\max }}\|\nabla u(\cdot, t)\|_{L^{2}}=+\infty,
$$

so it suffices to prove the bound (2.1). We will not keep track of the value of the constants $C_{q}$. It is possible to compute the value of the constant $C_{q}$ explicitly in terms of the sharp fractional Sobolev inequality in Theorem 2.2 , but we will not 
concern ourselves with long expressions for the value of the constant that would only clutter up this paper without adding any real mathematical insight.

First we will consider the case when $q=3, p=1$. Using the identity for enstrophy growth in Proposition 2.1, and applying Hölder's inequality and the Sobolev inequality, we find that for all $0<t<T_{\max }$, and for all $\lambda \in \mathbb{R}$,

$$
\begin{aligned}
\frac{\mathrm{d}}{\mathrm{d} t} \frac{1}{2}\|\nabla u(\cdot, t)\|_{L^{2}}^{2} & =-\|\Delta u\|_{L^{2}}^{2}-\langle-\Delta u-\lambda u,(u \cdot \nabla) u\rangle \\
& \leq\|-\Delta u-\lambda u\|_{L^{3}}\|u\|_{L^{6}}\|\nabla u\|_{L^{2}} \\
& \leq C\|-\Delta u-\lambda u\|_{L^{3}}\|\nabla u\|_{L^{2}}^{2} .
\end{aligned}
$$

Multiplying both sides by 2 and taking the infimum over $\lambda \in \mathbb{R}$, we find that

$$
\frac{\mathrm{d}}{\mathrm{d} t}\|\nabla u(\cdot, t)\|_{L^{2}}^{2} \leq C_{3} \inf _{\lambda \in \mathbb{R}}\|-\Delta u-\lambda u\|_{L^{3}}\|\nabla u\|_{L^{2}}^{2} .
$$

Applying Grönwall's inequality we find that for all $0<t<T_{\max }$

$$
\|\nabla u(\cdot, t)\|_{L^{2}}^{2} \leq\left\|\nabla u^{0}\right\|_{L^{2}}^{2} \exp \left(C_{3} \int_{0}^{t} \inf _{\lambda \in \mathbb{R}}\|-\Delta u-\lambda u\|_{L^{3}} \mathrm{~d} \tau\right),
$$

and we are done with the case where $q=3$.

Now we will consider the case $\frac{6}{5}<q<3$. First we will take $6<a<+\infty$ to be given by

$$
\frac{1}{a}=\frac{5}{18}-\frac{1}{3 q}
$$

and $2<b<6$ to be given by

$$
\frac{1}{b}=\frac{13}{18}-\frac{2}{3 q}
$$

Note that

$$
\frac{1}{a}+\frac{1}{b}+\frac{1}{q}=1
$$

so again using the identity for enstrophy growth from Lemma 2.1, Hölder's inequality, and the fractional Sobolev inequality, we find that for all $0<t<T_{\max }$ and for all $\lambda \in \mathbb{R}$,

$$
\begin{aligned}
\frac{\mathrm{d}}{\mathrm{d} t} \frac{1}{2}\|\nabla u(\cdot, t)\|_{L^{2}}^{2} & =-\|\Delta u\|_{L^{2}}^{2}-\langle-\Delta u-\lambda u,(u \cdot \nabla) u\rangle \\
& \leq-\|\Delta u\|_{L^{2}}^{2}+\|-\Delta u-\lambda u\|_{L^{q}}\|u\|_{L^{a}}\|\nabla u\|_{L^{b}} \\
& \leq-\|\Delta u\|_{L^{2}}^{2}+C\|-\Delta u-\lambda u\|_{L^{q}}\|\nabla u\|_{\dot{H}^{\alpha}}\|\nabla u\|_{\dot{H}^{\beta}},
\end{aligned}
$$

where

and

$$
\alpha=\frac{1}{2}-\frac{3}{a},
$$

$$
\beta=\frac{3}{2}-\frac{3}{b} .
$$

Plugging back into (2.2) and (2.3), we find that

$$
\alpha=\frac{1}{q}-\frac{1}{3},
$$

and

$$
\beta=\frac{2}{q}-\frac{2}{3} .
$$


It is straightforward to see that $0<\alpha<\frac{1}{2}$ and $0<\beta<1$, so we can interpolate between $L^{2}$ and $\dot{H}^{1}$ to find that

$$
\begin{aligned}
\frac{\mathrm{d}}{\mathrm{d} t} \frac{1}{2}\|\nabla u(\cdot, t)\|_{L^{2}}^{2} & \leq-\|\Delta u\|_{L^{2}}^{2}+C\|-\Delta u-\lambda u\|_{L^{q}}\|\nabla u\|_{L^{2}}^{2-(\alpha+\beta)}\|\nabla u\|_{\dot{H}^{1}}^{\alpha+\beta} \\
& =-\|\Delta u\|_{L^{2}}^{2}+C\|-\Delta u-\lambda u\|_{L^{q}}\|\nabla u\|_{L^{2}}^{3-\frac{3}{q}}\|-\Delta u\|_{L^{2}}^{\frac{3}{q}-1}
\end{aligned}
$$

where we have used the fact that $\alpha+\beta=\frac{3}{q}-1$. Recalling that $\frac{2}{p}=3-\frac{3}{q}$, we can see that $\frac{6}{5}<q<3$ implies that $1<p<4$. Take $\frac{4}{3}<r<+\infty$ to be the conjugate of $\mathrm{p}$.

$$
\frac{1}{p}+\frac{1}{r}=1
$$

Observe that

$$
\frac{2}{r}=2-\frac{2}{p}=\frac{3}{q}-1
$$

Using this to simplify and applying Young's inequality with exponents $r$ and $p$, we find that

$$
\begin{aligned}
\frac{\mathrm{d}}{\mathrm{d} t} \frac{1}{2}\|\nabla u(\cdot, t)\|_{L^{2}}^{2} & \leq-\|\Delta u\|_{L^{2}}^{2}+C\|-\Delta u\|_{L^{2}}^{\frac{2}{r}}\|-\Delta u-\lambda u\|_{L^{q}}\|\nabla u\|_{L^{2}}^{\frac{2}{p}} \\
& \leq C\|-\Delta u-\lambda u\|_{L^{q}}^{p}\|\nabla u\|_{L^{2}}^{2}
\end{aligned}
$$

Multiplying both sides by 2 and taking the infimum over $\lambda \in \mathbb{R}$, we find that

$$
\frac{\mathrm{d}}{\mathrm{d} t}\|\nabla u(\cdot, t)\|_{L^{2}}^{2} \leq C_{q} \inf _{\lambda \in \mathbb{R}}\|-\Delta u-\lambda u\|_{L^{q}}^{p}\|\nabla u\|_{L^{2}}^{2}
$$

Applying Grönwall's inequality we find that for all $0<t<T_{\max }$

$$
\|\nabla u(\cdot, t)\|_{L^{2}}^{2} \leq\left\|\nabla u^{0}\right\|_{L^{2}}^{2} \exp \left(C_{q} \int_{0}^{t} \inf _{\lambda \in \mathbb{R}}\|-\Delta u-\lambda u\|_{L^{q}}^{p} \mathrm{~d} \tau\right) .
$$

This completes the proof.

We will note here that the key element of the proof is the fact that

$$
\langle(u \cdot \nabla) u, u\rangle=0 .
$$

Because Tao's averaged 3D Navier-Stokes model equation also has the property

$$
\langle\tilde{B}(u, u), u\rangle=0
$$

the regularity criterion in Theorem 2.3 and the subsequent corollaries will also apply to Tao's model equation, for which there is finite-time blowup [26].

For general $\frac{6}{5}<q \leq 3$ we cannot compute

$$
\inf _{\lambda \in \mathbb{R}}\|-\Delta u-\lambda u\|_{L^{q}}
$$

explicitly, but in the special case where $q=2$, we can compute this infimum explicitly by making use of the Hilbert space structure.

Proposition 2.4. For all $u \in H^{2}\left(\mathbb{R}^{3}\right), u$ not identically zero,

$$
\inf _{\lambda \in \mathbb{R}}\|-\Delta u-\lambda u\|_{L^{2}}^{2}=\|-\Delta u\|_{L^{2}}^{2}\left(1-\frac{\|\nabla u\|_{L^{2}}^{4}}{\|u\|_{L^{2}}^{2}\|-\Delta u\|_{L^{2}}^{2}}\right)
$$


Proof. Fix $u \in H^{2}$. Define $f: \mathbb{R} \rightarrow \mathbb{R}$, by

$$
\begin{aligned}
f(\lambda) & =\|-\Delta u-\lambda u\|_{L^{2}}^{2} \\
& =\|-\Delta u\|_{L^{2}}^{2}-2\langle-\Delta u, u\rangle \lambda+\|u\|_{L^{2}}^{2} \lambda^{2} \\
& =\|-\Delta u\|_{L^{2}}^{2}-2\|\nabla u\|_{L^{2}}^{2} \lambda+\|u\|_{L^{2}}^{2} \lambda^{2}
\end{aligned}
$$

Taking the derivative of $f$ we find that

$$
f^{\prime}(\lambda)=-2\|\nabla u\|_{L^{2}}^{2}+2\|u\|_{L^{2}}^{2} \lambda .
$$

Let $\lambda_{0}=\frac{\|\nabla u\|_{L^{2}}^{2}}{\|u\|_{L^{2}}^{2}}$, and observe that $f$ has a global minimum at $\lambda_{0}$. Therefore we can compute that

$$
\begin{aligned}
\inf _{\lambda \in \mathbb{R}}\|-\Delta u-\lambda u\|_{L^{2}}^{2} & =\inf _{\lambda \in \mathbb{R}} f(\lambda) \\
& =f\left(\lambda_{0}\right) \\
& =\|-\Delta u\|_{L^{2}}^{2}-\frac{\|\nabla u\|_{L^{2}}^{4}}{\|u\|_{L^{2}}^{2}} \\
& =\|-\Delta u\|_{L^{2}}^{2}\left(1-\frac{\|\nabla u\|_{L^{2}}^{4}}{\|u\|_{L^{2}}^{2}\|-\Delta u\|_{L^{2}}^{2}}\right) .
\end{aligned}
$$

This completes the proof.

Using this identity for the infimum in the case where $q=2$, we will now prove Corollary [1.3, which is restated here for the reader's convenience.

Corollary 2.5. Suppose $u \in C\left(\left[0, T_{\max }\right) ; H^{1}\right)$ is a mild solution of the NavierStokes equation. Then for all $0<t<T_{\max }$

$$
\|\nabla u(\cdot, t)\|_{L^{2}}^{2} \leq\left\|\nabla u^{0}\right\|_{L^{2}}^{2} \exp \left(C_{2} \int_{0}^{t}\|-\Delta u\|_{L^{2}}^{\frac{4}{3}}\left(1-\frac{\|\nabla u\|_{L^{2}}^{4}}{\|u\|_{L^{2}}^{2}\|-\Delta u\|_{L^{2}}^{2}}\right)^{\frac{2}{3}} \mathrm{~d} \tau\right),
$$

where $C_{2}>0$ is taken as in Theorem 2.3. In particular, if $T_{\max }<+\infty$ then

$$
\int_{0}^{T_{\max }}\|-\Delta u\|_{L^{2}}^{\frac{4}{3}}\left(1-\frac{\|\nabla u\|_{L^{2}}^{4}}{\|u\|_{L^{2}}^{2}\|-\Delta u\|_{L^{2}}^{2}}\right)^{\frac{2}{3}} \mathrm{~d} t=+\infty .
$$

Proof. We will begin by observing that when $q=2, p=\frac{4}{3}$, then

$$
\frac{2}{p}+\frac{3}{q}=3
$$

Next we know from Proposition 2.4, that

$$
\inf _{\lambda \in \mathbb{R}}\|-\Delta u-\lambda u\|_{L^{2}}^{2}=\|-\Delta u\|_{L^{2}}^{2}\left(1-\frac{\|\nabla u\|_{L^{2}}^{4}}{\|u\|_{L^{2}}^{2}\|-\Delta u\|_{L^{2}}^{2}}\right) .
$$

Taking both sides of the equation to the $\frac{2}{3}$ power, we find that

$$
\inf _{\lambda \in \mathbb{R}}\|-\Delta u-\lambda u\|_{L^{2}}^{\frac{4}{3}}=\|-\Delta u\|_{L^{2}}^{\frac{4}{3}}\left(1-\frac{\|\nabla u\|_{L^{2}}^{4}}{\|u\|_{L^{2}}^{2}\|-\Delta u\|_{L^{2}}^{2}}\right)^{\frac{2}{3}},
$$

and then the result follows as an immediate corollary of Theorem 2.3 . 
Remark 2.6. It is worth considering in a little more detail the behaviour of the infimum in Theorem 2.3 in the presence of a possible finite-time blowup. In Proposition 2.4, we showed that that

This implies that

$$
\underset{\lambda \in \mathbb{R}}{\operatorname{arginf}}\|-\Delta u-\lambda u\|_{L^{2}}^{2}=\frac{\|\nabla u\|_{L^{2}}^{2}}{\|u\|_{L^{2}}^{2}} .
$$

$$
\begin{aligned}
\|\nabla u(\cdot, t)\|_{L^{2}}^{2} & =\underset{\lambda \in \mathbb{R}}{\arg \inf }\|(-\Delta u-\lambda u)(\cdot, t)\|_{L^{2}}^{2}\|u(\cdot, t)\|_{L^{2}}^{2} \\
& \leq \underset{\lambda \in \mathbb{R}}{\arg \inf }\|(-\Delta u-\lambda u)(\cdot, t)\|_{L^{2}}^{2}\left\|u^{0}\right\|_{L^{2}}^{2} .
\end{aligned}
$$

Therefore, if $T_{\max }<+\infty$, then

$$
\lim _{t \rightarrow T_{\max }} \underset{\lambda \in \mathbb{R}}{\arg \inf }\|(-\Delta u-\lambda u)(\cdot, t)\|_{L^{2}}^{2}=+\infty .
$$

While we cannot explicitly compute the infimum in Theorem 2.3, for $2<q<3$, we can compute this infimum in the Hilbert space $\dot{H}^{\beta} \hookrightarrow L^{q}$, using the inner product structure, and this will give us an explicit, scale-critical regularity criterion, albeit one requiring a higher degree of regularity.

Proposition 2.7. Suppose $0 \leq \beta<\frac{3}{2}$. For all $u \in \dot{H}^{\beta}\left(\mathbb{R}^{3}\right) \cap \dot{H}^{2+\beta}\left(\mathbb{R}^{3}\right)$, u not identically zero,

$$
\inf _{\lambda \in \mathbb{R}}\|-\Delta u-\lambda u\|_{\dot{H}^{\beta}}^{2}=\|u\|_{\dot{H}^{2+\beta}}^{2}\left(1-\frac{\|u\|_{\dot{H}^{1+\beta}}^{4}}{\|u\|_{\dot{H}^{\beta}}^{2}\|u\|_{\dot{H}^{2+\beta}}^{2}}\right) .
$$

Proof. Fix $0 \leq \beta<\frac{3}{2}$, and $u \in \dot{H}^{\beta}\left(\mathbb{R}^{3}\right) \cap H^{2+\beta}\left(\mathbb{R}^{3}\right)$. Define $f: \mathbb{R} \rightarrow \mathbb{R}$, by

$$
\begin{aligned}
f(\lambda) & =\|-\Delta u-\lambda u\|_{\dot{H}^{\beta}}^{2} \\
& =\|-\Delta u\|_{\dot{H}^{\beta}}^{2}-2\langle-\Delta u, u\rangle_{\dot{H}^{\beta}} \lambda+\|u\|_{\dot{H}^{\beta}}^{2} \lambda^{2} \\
& =\|u\|_{\dot{H}^{2+\beta}}^{2}-2\|u\|_{\dot{H}^{1+\beta}}^{2} \lambda+\|u\|_{\dot{H}^{\beta}}^{2} \lambda^{2}
\end{aligned}
$$

Taking the derivative of $f$ we find that

$$
f^{\prime}(\lambda)=-2\|u\|_{\dot{H}^{1+\beta}}^{2}+2\|u\|_{\dot{H}^{\beta}}^{2} \lambda
$$

Let $\lambda_{0}=\frac{\|u\|_{H^{1+\beta}}^{2}}{\|u\|_{\dot{H}^{\beta}}^{2}}$, and observe that $f$ has a global minimum at $\lambda_{0}$. Therefore we can compute that

$$
\begin{aligned}
\inf _{\lambda \in \mathbb{R}}\|-\Delta u-\lambda u\|_{\dot{H}^{\beta}}^{2} & =\inf _{\lambda \in \mathbb{R}} f(\lambda) \\
& =f\left(\lambda_{0}\right) \\
& =\|u\|_{\dot{H}^{2+\beta}}^{2}-\frac{\|u\|_{\dot{H}^{1+\beta}}^{4}}{\|u\|_{\dot{H}^{\beta}}^{2}} \\
& =\|u\|_{\dot{H}^{2+\beta}}^{2}\left(1-\frac{\|u\|_{\dot{H}^{1+\beta}}^{4}}{\|u\|_{\dot{H}^{\beta}}^{2}\|u\|_{\dot{H}^{2+\beta}}^{2}}\right) .
\end{aligned}
$$

This completes the proof.

Using Proposition 2.7, and the Sobolev inequality corresponding to the embed$\operatorname{ding} \dot{H}^{\beta} \hookrightarrow L^{q}$, we will now prove Corollary [1.4, which is restated here for the reader's convenience. 
Corollary 2.8. Suppose $u \in C\left(\left[0, T_{\max }\right) ; H^{1}\right)$ is a mild solution of the NavierStokes equation, and suppose $2 \leq \alpha \leq \frac{5}{2}, \alpha=\frac{1}{2}+\frac{2}{p}$. Then for all $0<t<T_{\max }$

$$
\|\nabla u(\cdot, t)\|_{L^{2}}^{2} \leq\left\|\nabla u^{0}\right\|_{L^{2}}^{2} \exp \left(\tilde{C}_{\alpha} \int_{0}^{t}\|u\|_{\dot{H}^{\alpha}}^{p}\left(1-\frac{\|u\|_{\dot{H}^{\alpha-1}}^{4}}{\|u\|_{\dot{H}^{\alpha-2}}^{2}\|u\|_{\dot{H}^{\alpha}}^{2}}\right)^{\frac{p}{2}} \mathrm{~d} \tau\right),
$$

where $\tilde{C}_{\alpha}>0$ depends only on $\alpha$. In particular, if $T_{\max }<+\infty$ then

$$
\int_{0}^{T_{\max }}\|u\|_{\dot{H}^{\alpha}}^{p}\left(1-\frac{\|u\|_{\dot{H}^{\alpha-1}}^{4}}{\|u\|_{\dot{H}^{\alpha-2}}^{2}\|u\|_{\dot{H}^{\alpha}}^{2}}\right)^{\frac{p}{2}} \mathrm{~d} t=+\infty .
$$

Note that the scaling relation between $\alpha$ and $p$ can alternatively be expressed by

$$
p=\frac{2}{\alpha-\frac{1}{2}}
$$

Proof. In the case where $\alpha=2$, this is precisely the same statement as Corollary 2.5. So fix $2<\alpha \leq \frac{5}{2}$. Let $\beta=\alpha-2$, so we have $0<\beta \leq \frac{1}{2}$. Let $\frac{1}{q}=\frac{1}{2}-\frac{\beta}{3}$, so we have the Sobolev embedding $\dot{H}^{\beta}\left(\mathbb{R}^{3}\right) \hookrightarrow L^{q}\left(\mathbb{R}^{3}\right)$. Then we can see that

$$
\frac{3}{q}=\frac{3}{2}-\beta=\frac{7}{2}-\alpha \text {. }
$$

Likewise we know that

so we can conclude that

$$
\frac{2}{p}=\alpha-\frac{1}{2}
$$

$$
\frac{2}{p}+\frac{3}{q}=3
$$

We know from the Sobolev inequality and from Proposition 2.7 that

$$
\begin{aligned}
\inf _{\lambda \in \mathbb{R}}\|-\Delta u-\lambda u\|_{L^{q}}^{2} & \leq C \inf _{\lambda \in \mathbb{R}}\|-\Delta u-\lambda u\|_{\dot{H}^{\beta}}^{2} \\
& =C\|u\|_{\dot{H}^{2+\beta}}^{2}\left(1-\frac{\|u\|_{H^{1+\beta}}^{4}}{\|u\|_{\dot{H}^{\beta}}^{2}\|u\|_{\dot{H}^{2+\beta}}^{2}}\right) \\
& =\|u\|_{\dot{H}^{\alpha}}^{2}\left(1-\frac{\|u\|_{\dot{H}^{\alpha-1}}^{4}}{\|u\|_{\dot{H}^{\alpha-2}}^{2}\|u\|_{\dot{H}^{\alpha}}^{2}}\right)
\end{aligned}
$$

Taking both sides of the equation to the power of $\frac{p}{2}$ we find that

$$
\inf _{\lambda \in \mathbb{R}}\|-\Delta u-\lambda u\|_{L^{q}}^{p} \leq C\|u\|_{\dot{H}^{\alpha}}^{p}\left(1-\frac{\|u\|_{\dot{H}^{\alpha-1}}^{4}}{\|u\|_{\dot{H}^{\alpha-2}}^{2}\|u\|_{\dot{H}^{\alpha}}^{2}}\right)^{\frac{p}{2}},
$$

and we have already shown that $\frac{2}{p}+\frac{3}{q}=3$, so the result then follows as an immediate corollary of Theorem 2.3 .

We mentioned in the introduction that without the term $\left(1-\frac{\|u\|_{\dot{H}^{\alpha}-1}^{4}}{\|u\|_{\dot{H}^{\alpha-2}-2}^{2}\|u\|_{\dot{H}^{\alpha}}^{2}}\right)^{\frac{p}{2}}$, Corollary 1.4 is an immediate corollary of the a variant of the LadyzhenskayaProdi-Serrin regularity criterion, which states that for a smooth solution of the Navier-Stokes equation, if $T_{\max }<+\infty$ and we have $\frac{2}{p}+\frac{3}{q}=2, \frac{3}{2}<q \leq+\infty$, then

$$
\int_{0}^{T_{\max }}\|\nabla u\|_{L^{q}}^{p} \mathrm{~d} t=+\infty
$$


This means that if $T_{\max }<+\infty$, then for all $1 \leq \alpha<\frac{5}{2}, \alpha=\frac{1}{2}+\frac{2}{p}$,

$$
\int_{0}^{T_{\max }}\|u\|_{\dot{H}^{\alpha}}^{p} \mathrm{~d} t=\int_{0}^{T_{\max }}\|\nabla u\|_{\dot{H}^{\alpha-1}}^{p} \mathrm{~d} t \geq C \int_{0}^{T_{\max }}\|\nabla u\|_{L^{q}}^{p} \mathrm{~d} t=+\infty .
$$

What is new in Corollary 2.8 is the term

$$
\left(1-\frac{\|u\|_{\dot{H}^{\alpha-1}}^{4}}{\|u\|_{\dot{H}^{\alpha-2}}^{2}\|u\|_{\dot{H}^{\alpha}}^{2}}\right)^{\frac{p}{2}}
$$

which measures the deficit in the interpolation inequality for the embedding $\dot{H}^{\alpha-2} \cap$ $\dot{H}^{\alpha} \hookrightarrow \dot{H}^{\alpha-1}$, stated below.

Proposition 2.9. For all $\alpha>\frac{1}{2}$, we have the embedding $\dot{H}^{\alpha-2} \cap \dot{H}^{\alpha} \hookrightarrow \dot{H}^{\alpha-1}$, with for all $f \in \dot{H}^{\alpha-2} \cap \dot{H}^{\alpha}$,

$$
\|f\|_{\dot{H}^{\alpha-1}}^{2} \leq\|f\|_{\dot{H}^{\alpha-2}}\|f\|_{\dot{H}^{\alpha}}
$$

Proof. Fix $f \in \dot{H}^{\alpha-2} \cap \dot{H}^{\alpha}$. Using the fact that $(-\Delta)^{\frac{1}{2}}$ is self-adjoint, and applying Hölder's inequality we find that

$$
\begin{aligned}
\|f\|_{\dot{H}^{\alpha-1}}^{2} & =\left\|(-\Delta)^{\frac{\alpha}{2}-\frac{1}{2}} f\right\|_{L^{2}}^{2} \\
& =\left\langle(-\Delta)^{\frac{\alpha}{2}-1} f,(-\Delta)^{\frac{\alpha}{2}} f\right\rangle \\
& \leq\left\|(-\Delta)^{\frac{\alpha}{2}-1} f\right\|_{L^{2}}\left\|(-\Delta)^{\frac{\alpha}{2}} f\right\|_{L^{2}} \\
& =\|f\|_{\dot{H}^{\alpha-2}}\|f\|_{\dot{H}^{\alpha}} .
\end{aligned}
$$

This completes the proof.

We will note that this interpolation inequality is related to eigenfunctions of the Laplacian because the only inequality in this proof is Hölder's inequality in (2.5), which holds with equality if

$$
(-\Delta)^{\frac{\alpha}{2}} f=\lambda(-\Delta)^{\frac{\alpha}{2}-1} f
$$

which in turn would imply that

$$
-\Delta f=\lambda f,
$$

and therefore that $f$ is an eigenfunction of the Laplacian. This can happen on the torus when working with $f \in \dot{H}^{\alpha-1}\left(\mathbb{T}^{3}\right)$ - for example the function $f(x)=$ $\sin \left(2 \pi x_{1}\right)$, is an eigenfunction of the Laplacian in $f \in \dot{H}^{\alpha-1}\left(\mathbb{R}^{3}\right)$, with $-\Delta f=$ $4 \pi^{2} f$-but not on the whole space. Nonetheless, the sharp constant in Proposition 2.9 is 1 for $\dot{H}^{\alpha-2}\left(\mathbb{R}^{3}\right) \cap \dot{H}^{\alpha}\left(\mathbb{R}^{3}\right)$, even though, because are no eigenfunctions of the Laplacian in $\dot{H}^{\alpha-2}\left(\mathbb{R}^{3}\right) \cap \dot{H}^{\alpha}\left(\mathbb{R}^{3}\right)$, this constant is not attained. We will prove this by considering functions whose Fourier transforms are supported on an annulus in $\mathbb{R}^{3}$.

Proposition 2.10. Suppose $u \in \dot{H}^{\alpha-2} \cap \dot{H}^{\alpha}$, with $u$ not identically zero, and

$$
\operatorname{supp} \hat{u} \subset\left\{\xi \in \mathbb{R}^{3}: R_{1} \leq|\xi| \leq R_{2}\right\} \text {. }
$$

Then

$$
\frac{\|u\|_{\dot{H}^{\alpha-1}}^{2}}{\|u\|_{\dot{H}^{\alpha-2}}\|u\|_{\dot{H}^{\alpha}}} \geq \frac{R_{1}^{2}}{R_{2}^{2}}
$$


This condition can be stated equivalently as

$$
1-\frac{\|u\|_{\dot{H}^{\alpha-1}}^{4}}{\|u\|_{\dot{H}^{\alpha-2}}^{2}\|u\|_{\dot{H}^{\alpha}}^{2}} \leq 1-\frac{R_{1}^{4}}{R_{2}^{4}} .
$$

Proof. First we will observe that

$$
\operatorname{supp} \hat{u} \subset\left\{\xi \in \mathbb{R}^{3}: R_{1} \leq|\xi| \leq R_{2}\right\}
$$

implies that

$$
\begin{aligned}
\|u\|_{\dot{H}^{\alpha-1}}^{2} & =\int_{\mathbb{R}^{3}}\left(4 \pi^{2}|\xi|^{2}\right)^{\alpha-1}|\hat{u}(\xi)|^{2} \mathrm{~d} \xi \\
& =\int_{\mathbb{R}^{3}} 4 \pi^{2}|\xi|^{2}\left(4 \pi^{2}|\xi|^{2}\right)^{\alpha-2}|\hat{u}(\xi)|^{2} \mathrm{~d} \xi \\
& \geq 4 \pi^{2} R_{1}^{2} \int_{\mathbb{R}^{3}}\left(4 \pi^{2}|\xi|^{2}\right)^{\alpha-2}|\hat{u}(\xi)|^{2} \mathrm{~d} \xi \\
& =4 \pi^{2} R_{1}^{2}\|u\|_{\dot{H}^{\alpha-2}}^{2} .
\end{aligned}
$$

Likewise, the condition on the support of $\hat{u}(2.6)$ implies that

$$
\begin{aligned}
\|u\|_{\dot{H}^{\alpha}}^{2} & =\int_{\mathbb{R}^{3}}\left(4 \pi^{2}|\xi|^{2}\right)^{\alpha}|\hat{u}(\xi)|^{2} \mathrm{~d} \xi \\
& =\int_{\mathbb{R}^{3}} 16 \pi^{4}|\xi|^{4}\left(4 \pi^{2}|\xi|^{2}\right)^{\alpha-2}|\hat{u}(\xi)|^{2} \mathrm{~d} \xi \\
& \leq 16 \pi^{4} R_{2}^{4} \int_{\mathbb{R}^{3}}\left(4 \pi^{2}|\xi|^{2}\right)^{\alpha-2}|\hat{u}(\xi)|^{2} \mathrm{~d} \xi \\
& =16 \pi^{4} R_{2}^{4}\|u\|_{\dot{H}^{\alpha-2}}^{2}
\end{aligned}
$$

Putting together (2.7) and (2.8) we find that

$$
\begin{aligned}
\frac{\|u\|_{\dot{H}^{\alpha-1}}^{4}}{\|u\|_{\dot{H}^{\alpha-2}}^{2}\|u\|_{\dot{H}^{\alpha}}^{2}} & \geq \frac{16 \pi^{4} R_{1}^{4}\|u\|_{\dot{H}^{\alpha-2}}^{4}}{16 \pi^{4} R_{2}^{4}\|u\|_{\dot{H}^{\alpha-2}}^{4}} \\
& =\frac{R_{1}^{4}}{R_{2}^{4}}
\end{aligned}
$$

It then immediately follows that

$$
\frac{\|u\|_{\dot{H}^{\alpha-1}}^{2}}{\|u\|_{\dot{H}^{\alpha-2}}\|u\|_{\dot{H}^{\alpha}}} \geq \frac{R_{1}^{2}}{R_{2}^{2}},
$$

and

$$
1-\frac{\|u\|_{\dot{H}^{\alpha-1}}^{4}}{\|u\|_{\dot{H}^{\alpha-2}}^{2}\|u\|_{\dot{H}^{\alpha}}^{2}} \leq 1-\frac{R_{1}^{4}}{R_{2}^{4}} .
$$

This completes the proof.

Proposition 2.10 shows that the sharp constant for the interpolation inequality in Proposition 2.9 is in fact 1 , and so the term

$$
\left(1-\frac{\|u\|_{\dot{H}^{\alpha-1}}^{4}}{\|u\|_{\dot{H}^{\alpha-2}}^{2}\|u\|_{\dot{H}^{\alpha}}^{2}}\right)^{\frac{p}{2}}
$$


does indeed measure the deficit in this interpolation inequality. The sharp constant 1 cannot be attained, because this would require

$$
\operatorname{supp} \hat{u} \subset\left\{\xi \in \mathbb{R}^{3}:|\xi|=R\right\},
$$

which is a set of measure zero.

Furthermore, Corollary 2.8 and Proposition 2.10 show that finite-time blowup solutions cannot concentrate on arbitrarily narrow bands in Fourier space, supported between an inner radius of $R_{1}(t)$ and an outer radius of $R_{2}(t)$, with the ratio $\frac{R_{1}(t)}{R_{2}(t)} \rightarrow 1$ arbitrarily quickly as $t \rightarrow T_{\max }$ relative to the size of $\|u(\cdot, t)\|_{\dot{H}^{\alpha}}^{p}$. In particular we will prove the following result, which is Corollary 1.5, and is restated here for the reader's convenience.

Corollary 2.11. Suppose $u \in C\left(\left[0, T_{\max }\right) ; H^{1}\right)$ is a mild solution of the NavierStokes equation, and suppose for all $0<t<T_{\max }$

$$
\operatorname{supp} \hat{u}(t) \subset\left\{\xi \in \mathbb{R}^{3}: R_{1}(t) \leq|\xi| \leq R_{2}(t)\right\} .
$$

Let $2 \leq \alpha \leq \frac{5}{2}, \alpha=\frac{1}{2}+\frac{2}{p}$. Then for all $0<t<T_{\max }$

$$
\|\nabla u(\cdot, t)\|_{L^{2}}^{2} \leq\left\|\nabla u^{0}\right\|_{L^{2}}^{2} \exp \left(\tilde{C}_{\alpha} \int_{0}^{t}\|u\|_{\dot{H}^{\alpha}}^{p}\left(1-\frac{R_{1}(\tau)^{4}}{\left.R_{2}(\tau)\right)^{4}}\right)^{\frac{p}{2}} \mathrm{~d} \tau\right),
$$

where $\tilde{C}_{\alpha}>0$ depends only on $\alpha$. In particular, if $T_{\max }<+\infty$ then

$$
\int_{0}^{T_{\max }}\|u\|_{\dot{H}^{\alpha}}^{p}\left(1-\frac{R_{1}(t)^{4}}{\left.R_{2}(t)\right)^{4}}\right)^{\frac{p}{2}} \mathrm{~d} t=+\infty .
$$

Proof. This corollary follows immediately from Corollary 2.8 and Proposition 2.10.

Remark 2.12. We will note that for a solution $u$ of the Navier-Stokes equation satisfying the hypotheses of Corollary 2.11, if $T_{\max }<+\infty$, then clearly

$$
\lim _{t \rightarrow T_{\max }} R_{2}(t)=+\infty
$$

otherwise we will have

$$
\liminf _{t \rightarrow T_{\max }}\|\nabla u(\cdot, t)\|_{L^{2}} \leq 2 \pi\left\|u^{0}\right\|_{L^{2}} \liminf _{t \rightarrow T_{\max }} R_{2}(t)<+\infty,
$$

which contradicts the assumption that $T_{\max }<+\infty$. This means that Corollary 2.11 rules out concentration arbitrarily quickly on narrow bands in Fourier space in the sense that $\frac{R_{1}(t)}{R_{2}(t)} \rightarrow 1$, although it should be noted that these bands are not necessarily narrow in the sense that $R_{2}(t)-R_{1}(t) \rightarrow 0$, because $R_{2}(t) \rightarrow+\infty$.

\section{ACKNowledgments}

The author would like to thank the referees for their thorough reading of the manuscript and helpful suggestions, which have improved the clarity of this paper. 


\section{REFERENCES}

[1] Dallas Albritton, Blow-up criteria for the Navier-Stokes equations in non-endpoint critical Besov spaces, Anal. PDE 11 (2018), no. 6, 1415-1456, DOI 10.2140/apde.2018.11.1415. MR.3803715

[2] Dallas Albritton and Tobias Barker, Global weak Besov solutions of the Navier-Stokes equations and applications, Arch. Ration. Mech. Anal. 232 (2019), no. 1, 197-263, DOI 10.1007/s00205-018-1319-0. MR3916974

[3] J. T. Beale, T. Kato, and A. Majda, Remarks on the breakdown of smooth solutions for the 3-D Euler equations, Comm. Math. Phys. 94 (1984), no. 1, 61-66. MR763762

[4] Z. Bradshaw and Z. Grujić, Frequency localized regularity criteria for the 3D Navier-Stokes equations, Arch. Ration. Mech. Anal. 224 (2017), no. 1, 125-133, DOI 10.1007/s00205-0161069-9. MR 3609247

[5] Dongho Chae and Hi-Jun Choe, Regularity of solutions to the Navier-Stokes equation, Electron. J. Differential Equations (1999), No. 05, 7. MR1673067

[6] Jean-Yves Chemin and Ping Zhang, On the critical one component regularity for 3-D NavierStokes systems (English, with English and French summaries), Ann. Sci. Éc. Norm. Supér. (4) 49 (2016), no. 1, 131-167, DOI 10.24033/asens.2278. MR3465978

[7] Jean-Yves Chemin, Ping Zhang, and Zhifei Zhang, On the critical one component regularity for 3-D Navier-Stokes system: general case, Arch. Ration. Mech. Anal. 224 (2017), no. 3, 871-905, DOI 10.1007/s00205-017-1089-0. MR3621812

[8] Qionglei Chen and Zhifei Zhang, Space-time estimates in the Besov spaces and the Navier-Stokes equations, Methods Appl. Anal. 13 (2006), no. 1, 107-122, DOI 10.4310/MAA.2006.v13.n1.a6. MR2275874

[9] A. Cheskidov and R. Shvydkoy, A unified approach to regularity problems for the 3D NavierStokes and Euler equations: the use of Kolmogorov's dissipation range, J. Math. Fluid Mech. 16 (2014), no. 2, 263-273, DOI 10.1007/s00021-014-0167-4. MR3208714

[10] L. Escauriaza, G. A. Seregin, and V. Šverák, $L_{3, \infty}$-solutions of Navier-Stokes equations and backward uniqueness, Uspekhi Mat. Nauk 58 (2003), no. 2(350), 3-44. MR1992563

[11] Hiroshi Fujita and Tosio Kato, On the Navier-Stokes initial value problem. I, Arch. Rational Mech. Anal. 16 (1964), 269-315. MR0166499

[12] Isabelle Gallagher, Gabriel S. Koch, and Fabrice Planchon, Blow-up of critical Besov norms at a potential Navier-Stokes singularity, Comm. Math. Phys. 343 (2016), no. 1, 39-82, DOI 10.1007/s00220-016-2593-z. MR3475661

[13] A. Kolmogoroff, The local structure of turbulence in incompressible viscous fluid for very large Reynold's numbers, C. R. (Doklady) Acad. Sci. URSS (N.S.) 30 (1941), 301-305. MR0004146

[14] Hideo Kozono, Takayoshi Ogawa, and Yasushi Taniuchi, The critical Sobolev inequalities in Besov spaces and regularity criterion to some semi-linear evolution equations, Math. Z. 242 (2002), no. 2, 251-278, DOI 10.1007/s002090100332. MR1980623

[15] Hideo Kozono and Yukihiro Shimada, Bilinear estimates in homogeneous TriebelLizorkin spaces and the Navier-Stokes equations, Math. Nachr. 276 (2004), 63-74, DOI 10.1002/mana.200310213. MR2100048

[16] Igor Kukavica and Mohammed Ziane, Navier-Stokes equations with regularity in one direction, J. Math. Phys. 48 (2007), no. 6, 065203, 10, DOI 10.1063/1.2395919. MR 2337002

[17] O. A. Ladyženskaja, Uniqueness and smoothness of generalized solutions of Navier-Stokes equations (Russian), Zap. Naučn. Sem. Leningrad. Otdel. Mat. Inst. Steklov. (LOMI) 5 (1967), 169-185. MR0236541

[18] Jean Leray, Sur le mouvement d'un liquide visqueux emplissant l'espace (French), Acta Math. 63 (1934), no. 1, 193-248, DOI 10.1007/BF02547354. MR.1555394

[19] Elliott H. Lieb, Sharp constants in the Hardy-Littlewood-Sobolev and related inequalities, Ann. of Math. (2) 118 (1983), no. 2, 349-374, DOI 10.2307/2007032. MR.717827

[20] Xiaoyutao Luo, A Beale-Kato-Majda criterion with optimal frequency and temporal localization, J. Math. Fluid Mech. 21 (2019), no. 1, Paper No. 1, 16, DOI 10.1007/s00021-019-0411-z. MR 3902460

[21] A. Obukhoff, On the energy distribution in the spectrum of a turbulent flow, C. R. (Doklady) Acad. Sci. URSS (N.S.) 32 (1941), 19-21. MR0005852

[22] Giovanni Prodi, Un teorema di unicità per le equazioni di Navier-Stokes (Italian), Ann. Mat. Pura Appl. (4) 48 (1959), 173-182, DOI 10.1007/BF02410664. MR126088 
[23] James Serrin, On the interior regularity of weak solutions of the Navier-Stokes equations, Arch. Rational Mech. Anal. 9 (1962), 187-195, DOI 10.1007/BF00253344. MR 136885

[24] S. L. Sobolev, Some applications of functional analysis in mathematical physics, Translations of Mathematical Monographs, vol. 90, American Mathematical Society, Providence, RI, 1991. Translated from the third Russian edition by Harold H. McFaden; With comments by V. P. Palamodov. MR 1125990

[25] Giorgio Talenti, Best constant in Sobolev inequality, Ann. Mat. Pura Appl. (4) 110 (1976), 353-372, DOI 10.1007/BF02418013. MR.463908

[26] Terence Tao, Finite time blowup for an averaged three-dimensional Navier-Stokes equation, J. Amer. Math. Soc. 29 (2016), no. 3, 601-674, DOI 10.1090/jams/838. MR3486169

Department of Mathematics and Statistics, McMaster University, Hamilton, Ontario, Canada

Email address: emiller@msri.org 